\title{
El espacio virtual de intercambio de información sobre recursos humanos en Ciencia y Tecnología de América Latina y el Caribe Del CV Lattes al CvLAC
}

Rebecca de los Ríos

Asesora Regional de Investigaciones en Salud. Organización Panamericana de la Salud, Washington DC.

delosrir@paho.org

Paulo Henrique de Assis Santana

Coordinador General de Sistemas de Información del Consejo Nacional de Desarrollo Científico y Tecnológico de Brasil (CNPq)

phas@cnpq.br

\section{Resumen}

Se describen los principales problemas de los diferentes sistemas de información para la gestión de la ciencia y tecnología de los países de América Latina y el Caribe y se identifican los retos y las alternativas para promover un mayor intercambio entre países del continente. La creación de un espacio de intercambio de información de los recursos humanos que participan en los sistemas de ciencia y tecnología, a través de una metodología común denominada el CVLAC, se constituye en una respuesta a los problemas identificados. El CVLAC parte de la experiencia brasileña del CvLattes. Se describen los objetivos y resultados esperados del Proyecto del CvLAC, ejecutado con la participación de Brasil, Colombia, Cuba, Chile, México y Venezuela. Se describen los desarrollos y alternativas a partir de los datos existentes en los países participantes en el proyecto, iniciando con Colombia y Chile, destacándose las infinitas posibilidades que las nuevas tecnologías de información digital y los nuevos desarrollos, logrados en este campo, hacen posible en términos de intercambio de datos.

Palabras clave

Cooperación técnica; Integración de bases de datos, Herramientas para intercambio de información.

\section{Virtual Space of Information Exchange about Human Resources on Science and Technology in Latin America and the Caribbean From CV Lattes to CvLAC}

\footnotetext{
Abstract

This paper presents the main problems of the different information systems for science and technology management in Latin America and the Caribbean and identifies the challenges and alternatives to promote a greater exchange among countries of the Continent. The creation of a space of information exchange of the human resources, which are shared in science and technology systems, through a common methodology called CVLAC, is an answer to the identified problems. CVLAC starts from the Brazilian experience of CVLattes. The objectives and results expected by the Project of CVLAC are described and carried out by Brazil, Colombia, Cuba, Chile, Mexico and Venezuela. A description is presented about the advancements and alternatives from the data existing in those countries participating in the project, starting with Colombia and Chile, emphasizing the endless possibilities which the new technologies of digital information and the new developments obtained in this field make possible with reference to data exchange.

Keywords

Technical cooperation; Databases integration; Tools for information exchange.
}

La necesidad de creación del espacio de intercambio de información sobre CyT de América Latina y el Caribe

Los sistemas de información para la gestión de la ciencia y la tecnología han sido en general, sistemas diseñados para dar respuesta al día-día de la gestión operacional de los organismos de fomento a la investigación y desarrollo tecnológico. Todos los organismos para el fomento de la ciencia, cuentan con bases de datos que almacenan información de las personas (científicos, investigadores, becarios etc.), de proyectos de investigación y de instituciones científicas y de innovación tecnológica. Estas bases por lo general son sistemas no abiertos y han tenido una escasa utilización en la gestión de la ciencia y la tecnología (CyT); entendida ésta, como procesos a través de los cuales se definen y evalúan políticas para el desarrollo de la ciencia y la tecnología, se asignan y administran los recursos destinados a tales fines y se facilitan los procesos de colaboración, intercambio científico e innovación tecnológica en los ámbitos nacional e internacional. El acceso oportuno y pertinente de la información para la gestión de la ciencia y la tecnología en su dimensión global y sectorial, se constituye por tanto, en un objetivo prioritario de la gerencia institucional y sectorial. Cabe destacar además que dicho acceso es demandado no sólo por los gestores y decisores sino también, por la propia comunidad científica nacional e internacional.

Pese a los esfuerzos importantes realizados por los Organismos de Ciencia y Tecnología (ONCyTs), persisten problemas que comparten la mayoría de estos organismos. A modo de síntesis se mencionan ${ }^{1}$.

- Diferentes instrumentos de captura de datos (tanto de curriculum, proyectos, instituciones etc.) que recogen la misma información y que no tienen sistemas adecuados de almacenamiento. Este problema se confronta al interior de las propias dependencias de los Ministerios de Ciencia y Tecnología (MCT) y de los ONCYT's, como al interior del sistema académico y de instituciones complejas de investigación. En consecuencia, los usuarios de los sistemas de fomento a la ciencia y la tecnología, tienen 
que llenar diferentes formularios (bien sea en línea o en papel) para los mismos fines y además, tienen que hacerlo un número repetido de veces.

- Las bases de datos que se generan a partir de los diferentes formatos de captura sobre curricula de los investigadores, grupos de investigación, proyectos, instituciones, etc., tienen escasa o ninguna integración y comunicación entre ellas y con acceso parcial o ningún acceso público por la Internet.

- Como consecuencia de lo anterior hay un limitado aprovechamiento y utilización de la información que se sistematiza en los MCT y ONCYT's tanto para la gestión estratégica en CyT global y sectorial en salud, como para promover e incrementar la visibilidad y el intercambio y comunicación entre científicos e investigadores de América Latina y el Caribe.

La convergencia y el uso integrado de las tecnologías de comunicación, computación y de contenidos en formato digital, cuyo paradigma es la Internet, han contribuido en los años recientes para crear un nuevo ambiente de acceso, intercambio y promoción del conocimiento a una escala global $^{2}$. Estas transformaciones han ejercido una profunda influencia sobre la concepción y funcionamiento de los sistemas de información automatizados, especialmente aquellos orientados a la CyT. La mayoría de los ONCYT's han realizado inversiones importantes no solo para automatizar sus procesos de gestión interna sino para incrementar el acceso y la disponibilidad de la información sobre CyT en la Internet. Son los casos por ejemplo, del Sistema Integrado de Información en Ciencia y Tecnología (SIICyT) de México ${ }^{3}$ (http://www.siicyt.mx) y La Plataforma Lattes del CNPq de Brasil (http:// www.cnpq.br).

Sin embargo, el fenómeno de la explosión de la información por la Internet tanto en términos de cantidad como de calidad, ha traído como consecuencia la dificultad creciente de su identificación, acceso y utilización. Aparecen nuevos problemas que tienen su impacto en la gestión de la actividad científica y tecnológica, sobre todo de los países en desarrollo, donde la visibilidad, localización y acceso oportuno a la información disponible en el Internet es un problema crucial. En términos de la gestión en CyT tanto a nivel global como sectorial, el gran reto que se plantea es el de encontrar la información relevante para un dado problema y que la misma esté disponible en un tiempo oportuno. Las comunidades científicas de los países en desarrollo además de ser comunidades pequeñas, son altamente demandantes de

Ci. Inf., Brasília, v. 30, n. 3, p. 42-47, set./dez. 2001 recursos de información siendo las fuentes de información de los países desarrollados las que se ofrecen como alternativas visibles a los usuarios las cuales, además de tener políticas restrictivas de acceso, tampoco resuelven un número importante de sus consultas y necesidades.

La actividad científico-tecnológica de América Latina y el Caribe además de tener un peso relativamente pequeño en la comunidad científica internacional, su visibilidad es limitada y su acceso a través de la Internet no resulta fácil ${ }^{4}$. Se requiere por tanto de alternativas y soluciones que den respuesta, no solo a la disponibilidad de información sobre CyT de los países de la Región, sino también, a los problemas de su identificación y localización oportuna en función de las necesidades de los usuarios.

¿Qué alternativas se tienen para la solución de este tipo de problemas? ¿Cómo y con qué tipo de herramientas se podrían incrementar los flujos de información sobre la Ciencia y la Tecnología de América Latina y el Caribe? ¿Cómo incrementar el acceso, visibilidad y localización de los investigadores y su producción científica en la Red?

Desde el punto de vista del usuario la solución perfecta sería aquella, que le permitiese someter sus necesidades de información interactuando con el menor número de interfaces y de ser posible, solamente con una. El someter sus necesidades de información y tener la información de diferentes fuentes y de manera consolidada, es una de las demandas más sentida que tienen los usuarios de información en CyT.

El Proyecto del Currículum Vitae en Ciencia y Tecnología de América Latina y el Caribe, conocido bajo el nombre del CvLAC, es una de las respuestas concretas que se pondrá a la disposición de la comunidad científica internacional y el público en general, para incrementar el flujo de información sobre los recursos calificados de la región en CyT. El mismo implica una fuerte acción de integración regional en el espacio de la Biblioteca Virtual en Salud (BVS) bajo el liderazgo de la Organización Panamericana de la Salud (OPS)/ Centro Regional de Información en Ciencias de la Salud (BIREME), de los más importantes proveedores de contenidos y servicios de información para la gestión en CyT a nivel global y sectorial, tanto en los ámbitos nacional como internacional.

\section{¿Que es CvLAC y cuáles son sus objetivos?}

CvLAC es un espacio común de integración e intercambio de información de los curricula de todas aquellas personas que forman parte de los sistemas de ciencia y tecnología 
de los países que participan en dicho espacio. Es en consecuencia, la colección sistematizada del conocimiento, la experiencia y la producción científica de todas las personas que participan en actividades de investigación, innovación y desarrollo tecnológico disponible en la Internet en el espacio común de la Biblioteca Virtual sobre Ciencia y Salud (BVS-CyS). Inicialmente ha sido creado para la integración e intercambio de información de los países de la Región de América Latina y el Caribe, pero estará abierto, a la participación de todos aquellos sistemas de CyT que deseen hacer disponible en la red, la información de sus recursos humanos calificados en CyT. El CvLAC adopta la tecnología-metodología desarrollada por el CNPq/Brasi conocida bajo el nombre de Currículum Lattes ${ }^{5}$ y con la participación de los países participantes en el proyecto bajo el liderazgo de OPS/BIREME, dicha tecnología se desarrolla, se adapta y se viabiliza para su progresiva implantación y operación a escala regional e internacional.

\section{¿ Cuáles países participan en el proyecto del CvLAC?}

El proyecto del CvLAC incorpora en su fase piloto a seis países de la Región de América Latina y el Caribe (Brasil, Colombia, Cuba, Chile, México y Venezuela). El desarrollo del prototipo del CvLAC tiene dos fases, una de desarrollo y operación piloto de metodologías e instrumentos y otra, de operación propiamente tal de los sistemas y herramientas con la participación inicial de 6 países mencionados.

El proyecto del Prototipo inició su ejecución en el año 2000 y se estima su culminación a finales del 2002, fecha a partir de la cual, el CvLAC estará en plena operación y abierto a la participación de los países que deseen formar parte del mismo.

\section{El CvLAC persigue como objetivos}

- Ampliar y optimizar el flujo de información sobre los recursos humanos calificados en CyT de los países de América Latina y el Caribe, mediante el desarrollo y operación de una metodología única denominada la metodología CvLAC.

- Promover el desarrollo de interfaces y estudios que faciliten el acceso y recuperación selectiva de registros individuales del CvLAC, incluyendo su clasificación, jerarquización, agrupamiento, tabulación y presentación según diferentes criterios, con el fin de brindar insumos para los procesos de gestión en CyT.
- Incrementar la visibilidad y mejorar la localización de la producción científica y tecnológica de los recursos humanos calificados en CyT de los países de América Latina y el Caribe, con fines de facilitar el intercambio y la cooperación entre países y entre comunidades científicas y de innovación tecnológica.

\section{Tendrá como resultados esperados}

- Una metodología/formulario padrón de captura de datos curriculares con criterios y clasificadores internacionales en tres idiomas (español, inglés y portugués) para ser instalado en los computadores de los usuarios (formulario off-line, CvLAC), disponible tanto en el site de navegación del CvLAC de la BVS-CyS, como en los sites de los ONCYT's de los países que adopten dicho instrumento de captura.

- Una base de metadatos regional operando e interactuando con las bases de datos nacionales de los curricula de los países participantes en el proyecto y el protocolo de intercambio de datos XLM disponible.

- Una interfaz de acceso unificado a los usuarios operando en la red, con diferentes herramientas desarrolladas y disponibles a los usuarios $(E j .:$ herramienta de búsqueda textual, el web-viewer, herramientas de auto-links y de links con otras bases, herramienta del graficador, jerarquizador etc.).

- Un site de navegación del CvLAC operando en la red, con acceso a la interfaz de la base de metadatos regional y bases nacionales y a las herramientas de intercambio y migración de datos disponibles a los gerentes y administradores cooperativos del sistema regional. (protocolos XML, extractor etc.)

- Un programa de promoción y difusión del CvLAC funcionando y operando en la Región.

\section{Estrategia de implantación y actividades}

CvLAC como se ha mencionado, es una espacio de intercambio y articulación de los curricula de las personas vinculadas a los sistemas de CyT de todas las áreas de las ciencias, las tecnologías y las artes. El Proyecto se constituye en uno de los instrumentos de la cooperación técnica de la Coordinación de Investigaciones de la Organización Panamericana de la Salud en apoyo a la gestión de la actividad científica y el intercambio de investigadores y tiene como instancia de ejecución regional al Centro Regional de Información de Ciencias de la Salud (BIREME). En los países participantes en el 
desarrollo del prototipo, las instancias de coordinación y ejecución nacional del proyecto descansa en los Organismos de Fomento de la Ciencia y la Tecnología (ONCyTs).

La estrategia de implantación del CvLAC como proyecto de la Biblioteca Virtual sobre Ciencia y Salud, se concibe en dos fases cuyas actividades se ejecutan en períodos diferentes y en algunos casos de manera simultánea:

\section{Fase I}

Desarrollo y prueba piloto de herramientas: El instrumento de captura de datos, (formulario CvLAC); desarrollo piloto de los sistemas de envío y recepción de datos a través del Internet (ventanilla virtual) y desarrollo y operación piloto de la interfaz y herramientas para los usuarios. Esta fase contempla la realización de las siguientes actividades:

1. Desarrollo y prueba del formulario de captura de datos, denominado formulario CvLAC en su versión off-line.

2. Desarrollo y prueba de la ventanilla virtual de recepción de datos y de la interfaz piloto con las herramientas para los usuarios.

\section{Fase II}

Desarrollo y prueba de las herramientas para la migración de datos de los sistemas/bases nacionales y operación del espacio de intercambio de los curricula de los países participantes en el proyecto.

Para tales fines y bajo la conducción técnica de OPS/ BIREME en colaboración con el CNPq/Brasil, el Grupo Stela de la UFSC y el equipo de desarrolladores del CvLAC, se realizarán las siguientes actividades:

1. Misiones a los países participantes e interesados en participar en el espacio de intercambio CvLAC para establecer los convenios de intercambio de información de los curricula de los recursos humanos de los sistemas nacionales de CyT. (Diagnóstico de situación y estrategia de adopción de la metodología CvLAC).

2. Elaboración del protocolo de intercambio de datos en XML, creación de la comunidad virtual LMPLAC (Lenguaje de Marcación de la Plataforma de Información en CyT) y de las herramientas tecnológicas para el intercambio de datos (importación, extracción, exportación).
3. Establecimientos de convenios para la operación y mantenimiento del CvLAC a nivel regional y nacional.

Culminado el proceso de la prueba piloto y teniendo la versión definitiva del formulario CvLAC, se tendrán las bases metodológicas y técnicas a nivel regional para operar y mantener una interfaz única y una base regional de curricula de los recursos humanos participantes en los sistemas nacionales de CyT de los países de América Latina y el Caribe. Los países participantes en el proyecto y los que en el futuro se vayan adscribiendo al mismo, irán definiendo sus modelos de participación y las formas de intercambio e interconexión de sus sistemas heterogéneos, bajo una interfaz de acceso único construida para tales propósitos.

\section{Estado actual de desarrollo}

En el momento de publicación de este artículo, ya se ha cumplido con la Fase I y, para la fase II, ya se cuentan con los datos de Brasil, Chile y Colombia en una base de datos que puede ser consultada de forma independiente o integrada.

En la figura 1, se puede ver, en esquema, la integración de los sistemas de la fase I. El formulario CvLAC ya puede ser utilizado para enviar un currículo por Internet hasta una ventanilla virtual. Esta ventanilla recibe el currículo, lo carga en la base de datos y emite un recibo electrónico para el usuario. Enseguida, el currículo se hace disponible para búsqueda textual en Internet. Además de esto, el formulario CvLAC pude exportar e importar los currículos 
en formato XML (eXtended Markup Language). Así, el formulario se transforma en un instrumento universal para intercambio de datos.

En la Figura 2a, se puede ver la interfaz de búsqueda textual en su versión más sencilla (http://dwcvlac.cnpq.br: 12010/dcd_dwcvlac/owa/consulta pesq.inicio) La búsqueda se puede hacer recuperando currículos (personas) o producciones científicas (artículos, libros, etc.).

Si se hace la recuperación de currículos, mirándolos se puede ver la producción individual de cada persona. Si se hace la recuperación de productos, a través de cada uno de ellos se pude ver el currículo de la persona que lo ha informado.

En la Figura 2b, se puede ver un resultado de una búsqueda por currículos en que los nombres de las personas tienen banderitas al lado indicando su nacionalidad y hay punteros para el contenido de cada currículo.

Los currículos resultantes de una búsqueda se pueden ordenar por diversos criterios: número de publicaciones, número de patentes, número de tutorías, etc. Cuando se pulsa sobre ordenación, un formulario se hace presente para que se pueda escoger los criterios (figura 2.b).

En la fase II, los datos de Brazil, Chile y Colombia ya están disponibles y hay negociaciones en curso para inclusión de los datos de Mexico, Venezuela y Cuba.
FIGURA 2A

Interfaz de búsqueda

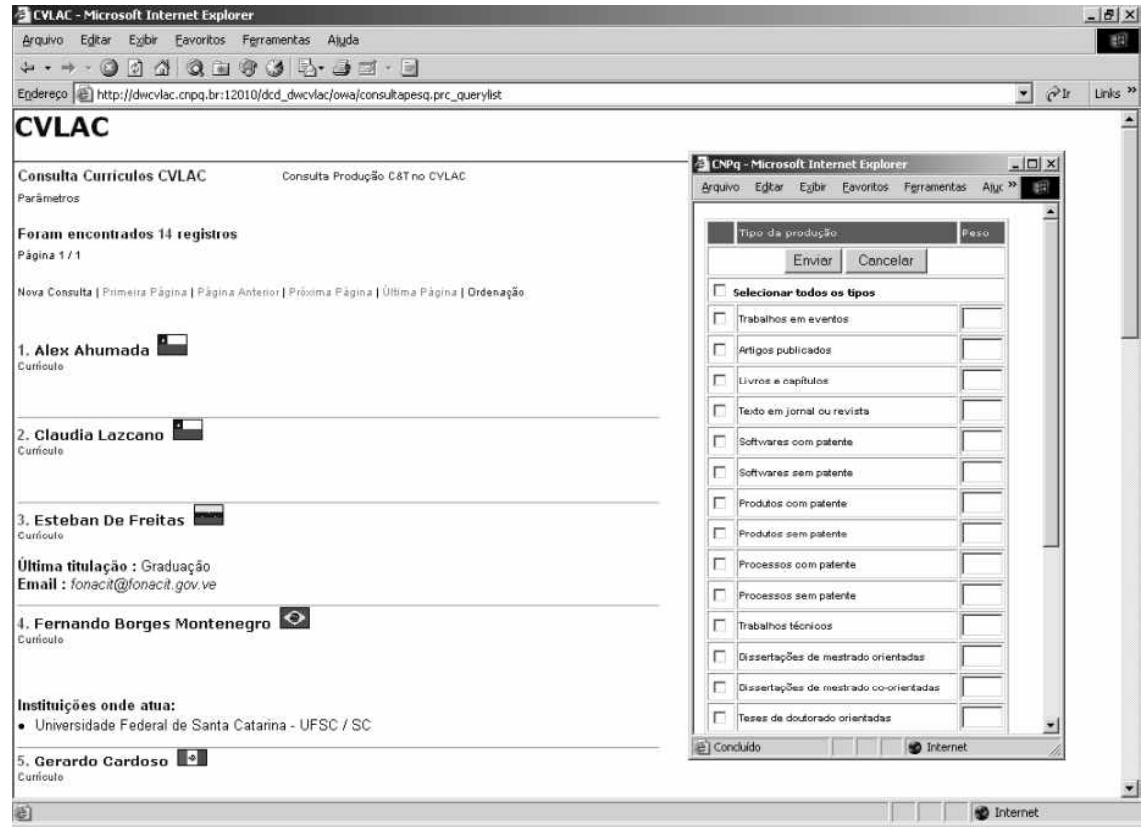

FIGURA 2B

Resultado de una búsqueda por currículos

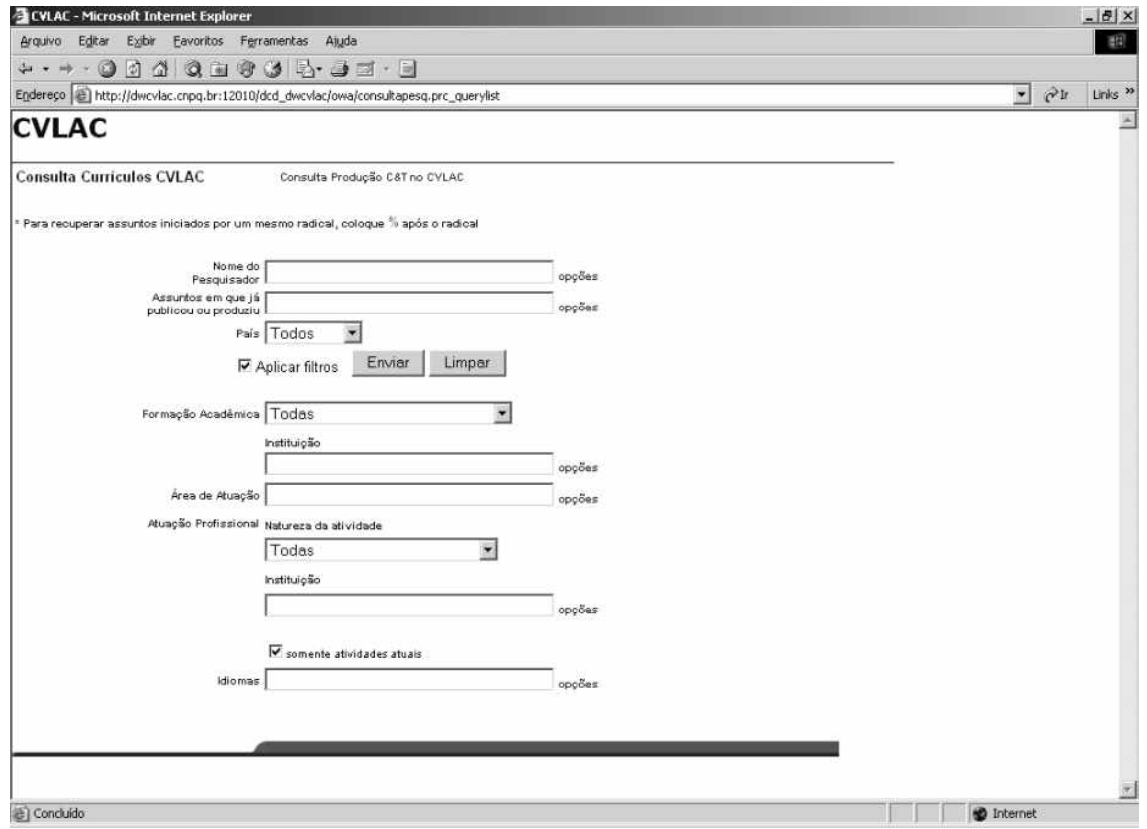


En la Figura 3a, se puede ver esquemáticamente la situación de la fase II. Para los datos de Chile, Colombia y Brasil, se puede hacer búsqueda individual, utilizando la interfaz de cada país, o búsqueda integrada, haciendo uso de la interfaz común.

La Figura $3 b$ presenta un ejemplo de búsqueda utilizando la interfaz integrada, con recuperación de especialistas de los tres países que ya enviaran sus datos.

\section{REFERENCIAS BIBLIOGRAFICAS}

1. Organización Panamericana de la Salud (OPS). Biblioteca Virtual en Ciencia y Salud (BVS CyS) Plataforma Internacional de Datos para la Gestión de la Ciencia y Tecnología. Coordinación de Investigaciones. Mimeo, 2001

2. Instituto Brasilero de Información en Ciencia y Tecnología (IBICT). Proyecto de la Biblioteca Digital Brasileira. Mimeo, 2001

3. Sistema Integrado de Información en Ciencia y Tecnología. Consejo Nacional de Ciencia y Tecnología de México, en www.conacyt.mx

4. Centro Regional de Información en Ciencias de la Salud (BIREME). La Biblioteca Virtual en Salud (BVS), en www.bireme.org

5. Conselho Nacional de Desenvolvimento Científico e Tecnológico ( $\mathrm{CNPq})$. Curriculum Lattes Versión 4.0 en www.cnpq.br

Artigo recebido em 04/12/2001

Ci. Inf., Brasília, v. 30, n. 3, p. 42-47, set./dez. 2001
FIGURA 3B

Resultado de búsqueda hecha con la interfaz integrada (Fase II)

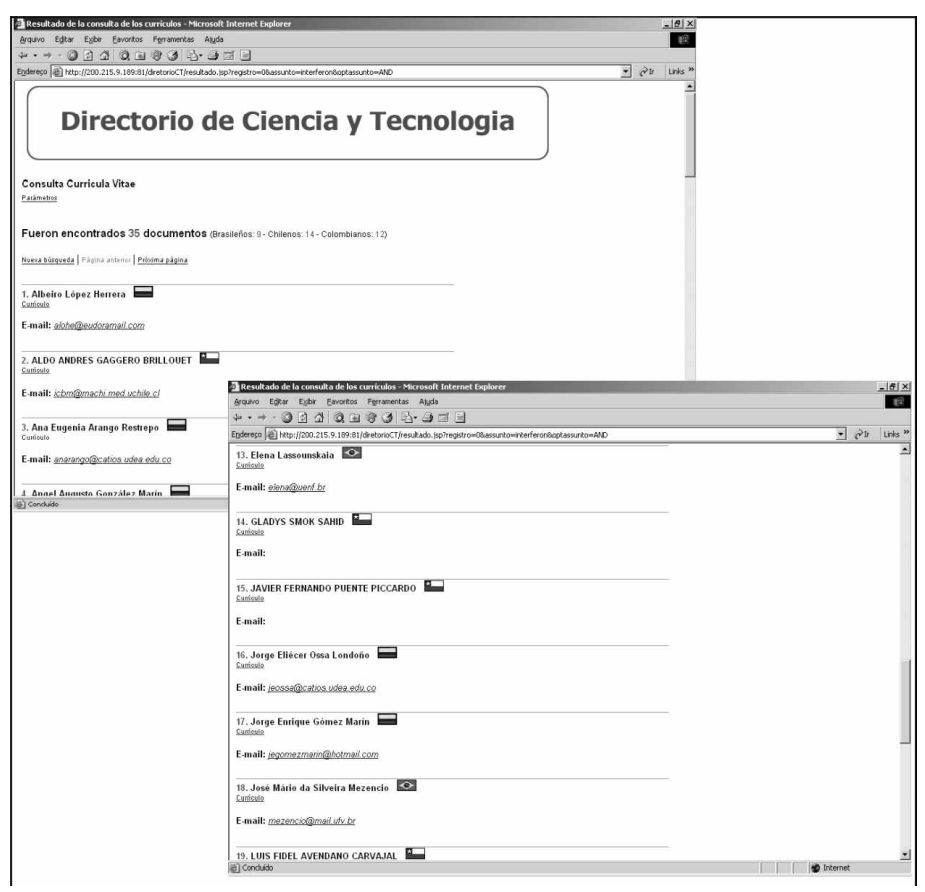

FIGURA 3A

Curriculum Vitae en Ciencia y Tecnología (Fase II)

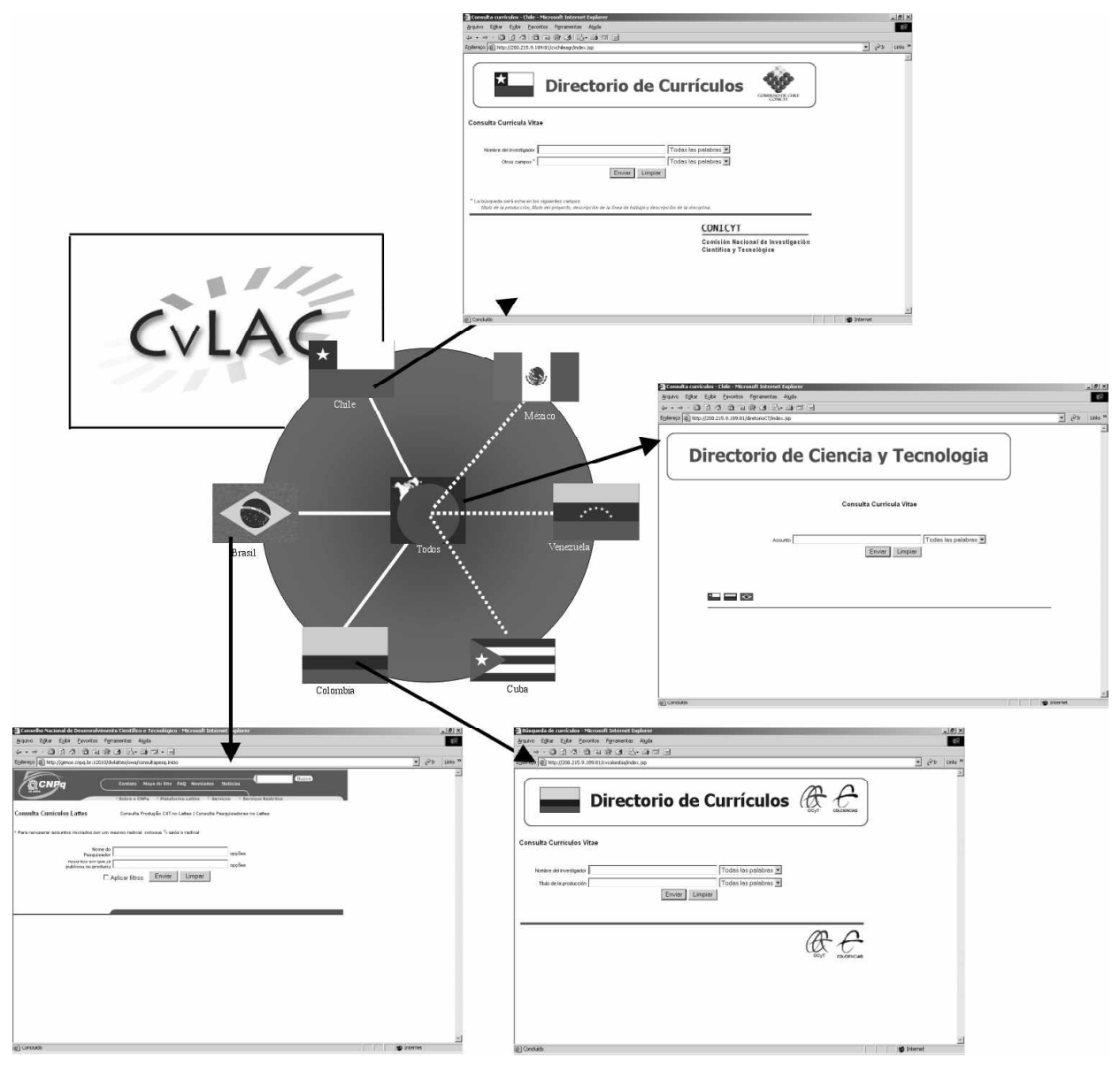

